\title{
Interval Identification of FMR Parameters for Spin Reorientation Transition in (Ga,Mn)As
}

M.W. Gutowski ${ }^{a *}$, W. Stefanowicz ${ }^{a}$, O. Proselkov ${ }^{a}$, M. SAWiCki $^{a}$, R. ŻUBEREK ${ }^{a}$ AND J. SADOWSKI ${ }^{a, b}$

${ }^{a}$ Institute of Physics, Polish Academy of Sciences, al. Lotników 32/46, 02-668 Warszawa, Poland

${ }^{b}$ MAX-lab, Lund University, Lund, Sweden

In this work we report results of ferromagnetic resonance studies of a $6 \% 15 \mathrm{~nm}$ (Ga,Mn)As layer, deposited on (001)-oriented GaAs. The measurements were performed with in-plane oriented magnetic field, in the temperature range between $5 \mathrm{~K}$ and $120 \mathrm{~K}$. We observe a temperature induced reorientation of the effective in-plane easy axis from [110] to [110] direction close to the Curie temperature. The behavior of magnetization is described by anisotropy fields, $H_{\text {eff }}\left(=4 \pi M-H_{2 \perp}\right), H_{2 \|}$, and $H_{4 \|}$. In order to precisely investigate this reorientation, numerical values of anisotropy fields have been determined using powerful — but still largely unknown - interval calculations. In simulation mode this approach makes possible to find all the resonance fields for arbitrarily oriented sample, which is generally intractable analytically. In "fitting" mode we effectively utilize full experimental information, not only those measurements performed in special, distinguished directions, to reliably estimate the values of important physical parameters as well as their uncertainties and correlations.

PACS: 07.05.Kf, 68.47.Fg, 75.30.Gw, 75.50.Pp, 75.70.-i, 75.70.Ak, 75.70.Cn, 75.70.Rf, 76.50.+g

\section{Motivation}

Despite numerous and intensive studies, an origin of the in-plane uniaxial magnetic anisotropy in ( $\mathrm{Ga}, \mathrm{Mn}) \mathrm{As}$ remains unknown. However, both its strength and orientation can be described on the ground of $p-d$ Zener model assuming the existence of a fictitious epitaxial strain [1-3]. On the other hand, as this is the leading magnetic anisotropy at elevated temperatures and that the means of its control have already been demonstrated [4], further studies on this intriguing property are timely and important. In particular, a presence of the temperature-induced $90^{\circ}$ rotation of the direction of the easy axis [1] may ease the vector manipulation of magnetization in future devices. In this communication we report on the technical analysis and results of FMR studies of such a (Ga,Mn)As layer that exhibit the easy axis rotation at temperatures close to its Curie temperature.

The free energy density for our system, expressed by anisotropy fields ( $H$ 's) rather than by more customary anisotropy constants ( $K$ 's), has the form (the Zeeman term, $g \mu_{\mathrm{B}} H M$, and inactive out-of-plane fourfold anisotropy part have been omitted):

$$
\begin{aligned}
& F=2 \pi M^{2} \cos ^{2} \theta \\
& -\frac{1}{2} M H_{2 \perp} \cos ^{2} \theta \\
& -\frac{1}{2} M H_{2 \|} \sin ^{2} \theta \sin ^{2}\left(\varphi-\frac{\pi}{4}\right) \\
& -\frac{1}{16} M H_{4 \|}(3+\cos 4 \varphi) \sin ^{4} \theta,
\end{aligned}
$$

where (1) is shape anisotropy, (2) - ordinary uni-

\footnotetext{
* corresponding author; e-mail: marek.gutowski@ifpan.edu.pl
}

axial out-of-plane anisotropy, (3) - uniaxial in-plane anisotropy, and (4) - fourfold, in-plane anisotropy.

Here polar angles $\theta$ and $\varphi$ refer to the orientation of the magnetization vector, $\boldsymbol{M}$, not to the orientation of an external field $\boldsymbol{H}$.

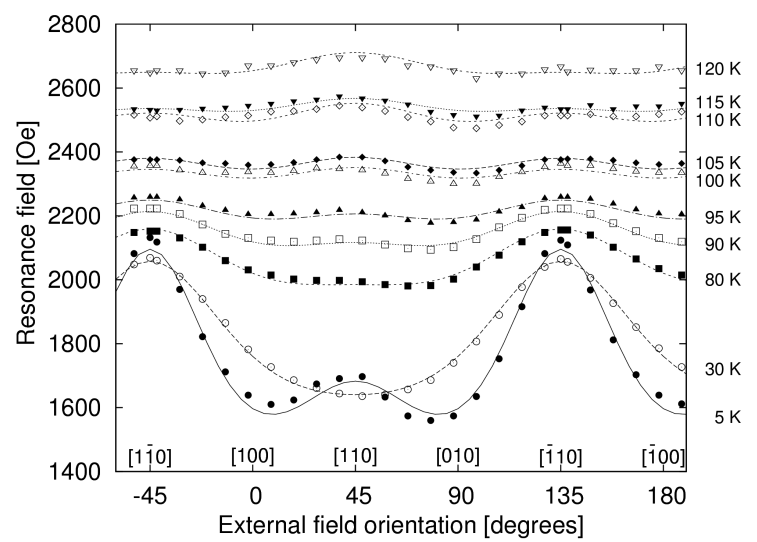

Fig. 1. Experimental values of FMR fields vs. external field orientation and temperature (points). Lines show computed spectra.

Original experimental data, taken at fixed frequency 9.378 GHz, are shown in Fig. 1, together with simulated spectra.

The numerical values of three parameters: $H_{\text {eff }}=$ $4 \pi M-H_{2 \perp}$ (where $\left.H_{2 \perp} \equiv 2 K_{2 \perp} / M\right), H_{2 \|}$ and $H_{4 \|}$ were determined for each temperature separately.

\section{Outline of numerical procedure}

We are using interval calculations not only for accurate simulations of FMR spectra when the values of all relevant parameters are known, but also to estimate ("fit") 
such parameters, together with their uncertainties and correlations, utilizing full information hidden in experimental data. An excellent introduction to interval arithmetics can be found in [5]. Here we limit ourselves to the very brief description: (i) an interval $\boldsymbol{x}$ is a bounded set of real numbers: $\boldsymbol{x} \equiv[a, b]=\{\mathbb{R} \ni x: a \leqslant x \leqslant b\}$, (ii) it is possible to perform arithmetic operations, evaluate functions, etc., using intervals instead of numbers, (iii) the so-obtained results are intervals as well, (iv) the multidimensional intervals are called boxes for obvious reasons, and (v) ordinary real numbers may be identified with ("thin") intervals, for example $7=[7,7]$. Similarly, $f(\boldsymbol{x})$ is an interval containing all the possible results of evaluation of $f(x)$ when $x \in \boldsymbol{x}$. Unfortunately, $f(\boldsymbol{x})$ usually overestimates the range of true values of $f(x)$ - but always contains them all.

The unconventionality of our approach to fixed frequency FMR data fitting is that we try to adjust unknown parameters in such a way that the classical formula

$$
\left(\frac{\omega}{g \mu_{\mathrm{B}}}\right)^{2}=\frac{1}{(M \sin \theta)^{2}}\left[\frac{\partial^{2} F}{\partial \theta^{2}} \frac{\partial^{2} F}{\partial \varphi^{2}}-\left(\frac{\partial^{2} F}{\partial \theta \partial \varphi}\right)^{2}\right]
$$

is satisfied for each experimental datum.

The main difficulty lies in the fact that the partial derivatives of free energy density have to be evaluated at (stable) equilibrium position of magnetization vector, characterized by two (initially unknown) polar angles $\varphi$ and $\theta$, and being the solution (s) of the equation's system

$$
\nabla F=(\partial F / \partial \theta, \partial F / \partial \varphi)=0 .
$$

This makes the inversion of formula (5), to obtain resonance field(s) as a function of microwave frequency $\omega$, essentially impossible.

Our algorithm operates on a list $\mathcal{L}$ of boxes. At the beginning, the list contains only one member - the initial search domain. Further we perform following steps:

1. select the biggest box from the list $\mathcal{L}$;

2. bisect its longest edge obtaining two offspring boxes, then remove the parent box from the list $\mathcal{L}$;

3. investigate each one of the two offspring and:

- discard it, if infeasible, or

- put it back on the list $\mathcal{L}$, otherwise.

The above procedure is repeated until the list contains only small boxes. The rest is easy, provided the final cluster of boxes consists of only one connected component. For details on how to calculate mean values, variances and correlations between the searched parameters see [6]. Here we only clarify when the box is considered infeasible. First of all, each box is characterized by its quality factor, computed as an interval quantity $Q=[\underline{Q}, \bar{Q}]$. For each resonance field (and its corresponding orientation) we try to evaluate the interval value of right hand side (r.h.s.) of expression (5). If the upper bound of so calculated interval is negative, then the currently considered box of searched parameters is infeasible as it cannot describe any resonance at all. If all experimental data pass this test, then the ranges of their resonance frequencies can be found from (5) and the interval $\boldsymbol{Q}$ can be evaluated. Again, for each data element $i$ the absolute difference between both sides of (5) is calculated, producing the interval $\boldsymbol{\Delta} \omega_{i}$. Finally, $\boldsymbol{Q}=\max _{i} \boldsymbol{\Delta} \omega_{i}$ (other choices are possible but we prefer this one). The box is considered infeasible when its $\underline{Q}$ exceeds the reference value, which is equal to the lowest $\bar{Q}$ ever seen during calculations.

It remains to comment on resonance frequency calculation. The angles $(\theta, \varphi)$ are unknown and should be determined prior to resonance frequency evaluation, for each data element separately. Starting from full ignorance $(\theta \in[0, \pi], \varphi \in[0,2 \pi])$, we divide this initial $2 \mathrm{D}$ box until its edges are shorter than, say, $0.5^{\circ}$. Discarded are all boxes satisfying either of two conditions: $0 \notin \partial F / \partial \theta$ or $0 \notin \partial F / \partial \varphi$ - they certainly cannot contain the equilibrium position of the vector $\boldsymbol{M}$. Needless to say that failure to find such a position immediately invalidates the searched parameter box. Similarly, negative upper bound of $\partial^{2} F / \partial \theta^{2}$ (or $\partial^{2} F / \partial \varphi^{2}$ ) is another good reason to discard such a box.

\section{Results and discussion}

Following the procedure described in previous section, we have computed the values of parameters determining the free energy density (1)-(4). During computation $g$ was kept fixed at 2.00. The results are presented in Fig. 2. The numerical values are consistent with those reported by others [7-9], obtained by the same technique but at lower temperatures. Using those results, without any prior smoothing, we were able to determine the directions of spontaneous magnetization in the interesting temperature range, near $T_{\mathrm{C}}$.

In Fig. 3 one can see that, in absence of the external field, there are only two such directions, antiparallel to each other, not four as one might expect from symmetry arguments. The presence of external magnetic field, oriented along [110] (Earth's field is sufficient) breaks even this symmetry, and the spontaneous magnetization aligns itself exactly along the external field above the transition temperature. Below the transition temperature the spontaneous magnetization deviates considerably from its "natural" [110] (or, equivalently, [110]) position. This means that precise examination of spontaneous magnetization, especially of its components perpendicular to the external field, is difficult. The temperature range, in which the reorientation occurs, is also very sensitive to the presence of even very weak field.

The behavior of anisotropy fields near $T_{\mathrm{C}}$, estimated as $\approx 113 \mathrm{~K}$ for our sample, is somewhat unusual: they all should go to 0 as $T \rightarrow T_{\mathrm{C}}$ - but they do not. In addition, the simulated angular FMR dependences (Fig. 1) seem not so accurate as one might expect. Those intriguing facts, together with high reliability of interval analysis, strongly suggest that the uniaxial in-plane anisotropy 


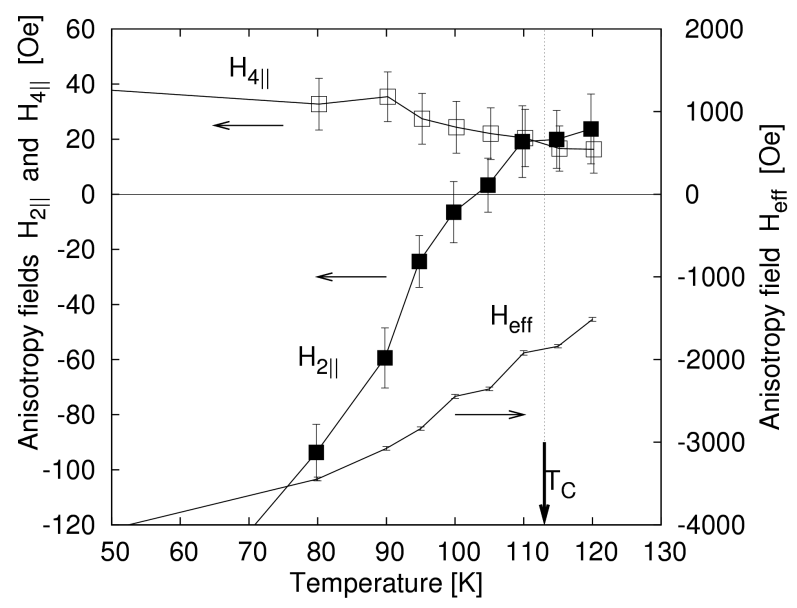

Fig. 2. Anisotropy fields vs. temperature - computed values. Lines are eye-guides only. Apparent lack of smoothness may be attributed to inaccurate temperature readings (the measurements were taken during two separate sessions). For better visibility, the curves $H_{2 \|}$ and $H_{4 \|}$ are shifted horizontally by $-0.2 \mathrm{~K}$ and $+0.2 \mathrm{~K}$, respectively.

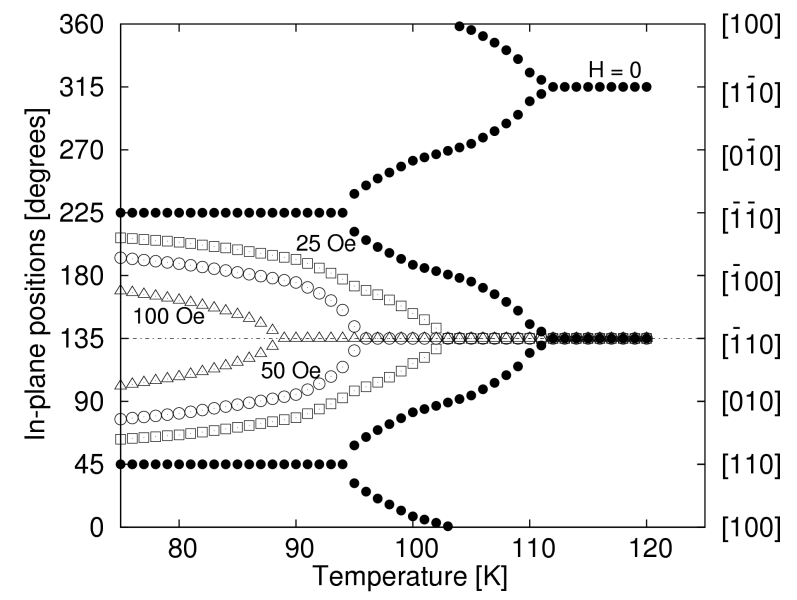

Fig. 3. Equilibrium position of magnetization vector vs. temperature for various strengths of the external field. The field is always directed along high-temperature easy axis [110], marked also as $135^{\circ}$, and its strength is as indicated.

may be incorrectly accounted for in (1)-(4). Since the samples exhibiting this special behavior are very thin, in $\mathrm{nm}$ range, then it is quite possible that the source of uniaxial anisotropy may be related to surface effects. Indeed, AFM surface studies of MBE-grown samples [10] revealed the presence of well ordered ripples, parallel to [110] direction. If this was true, then the free energy expression should be appended with the surface term [11], proportional to $|\cos (\varphi-\pi / 4)|$. This, however, is beyond the scope of a current paper.

\section{Conclusions}

The interval calculus has been demonstrated to be a very powerful tool for difficult problems of experimental data analysis. In particular, it is probably the only method able to utilize complete experimental information acquired during FMR measurements. Its unrivalled reliability allows us to state a sound hypothesis concerning the nature of the somewhat mysterious in-plane uniaxial magnetic anisotropy observed in thin layers of (Ga,Mn)As grown on (001)-oriented GaAs substrate.

We have also shown that FMR technique is very helpful in accurate tracing the temperature dependence of magnetic anisotropy in close vicinity of the Curie temperature, that is where the magnetometric data are least reliable.

Last but not least: the reliability is costly. The time complexity of interval methods grows exponentially with the number of estimated parameters, so it is very important to eliminate "bad" boxes as early as possible.

\section{Acknowledgments}

This work was supported in part by EC Network SemiSpinNet (PITN-GA-2008-215368) and Polish MNiSW 2048/B/H03/2008/34 grant.

\section{References}

[1] M. Sawicki, K.-Y. Wang, K.W. Edmonds, R.P. Campion, C.R. Staddon, N.R.S. Farley, C.T. Foxon, E. Papis, E. Kamińska, A. Piotrowska, T. Dietl, B.L. Gallagher, Phys. Rev. B 71, 121302(R) (2005).

[2] J. Zemen, J. Kučera, K. Olejnik, T. Jungwirth, Phys. Rev. B 80, 155203 (2009).

[3] W. Stefanowicz, C. Śliwa, P. Aleshkevych, T. Dietl, M. Döppe, U. Wurstbauer, W. Wegscheider, D. Weiss, M. Sawicki, Phys. Rev. B 81, 155203 (2010).

[4] D. Chiba, M. Sawicki, Y. Nishitani, Y. Nakatani, F. Matsukura, H. Ohno, Nature (London) 455, 515 (2008).

[5] http://www.cs.utep.edu/interval-comp/ .

[6] M.W. Gutowski, arxiv/\hz0903.0365.

[7] X. Liu, J.K. Furdyna, J. Phys., Condens. Matter 18, R245 (2006).

[8] Y.Y. Zhou, X. Liu, J.K. Furdyna, M.A. Scarpulla, O.D. Dubon, Phys. Rev. B 80, 224403 (2009).

[9] X. Liu, Y. Sasaki, J.K. Furdyna, Phys. Rev. B 67, 205204 (2003).

[10] S. Piano, X. Marti, A.W. Rushforth, K.W. Edmonds, R.P. Campion, O. Caha, T.U. Schulli, V. Holý, B.L. Gallagher, Appl. Phys. Lett. 98, 152503 (2011).

[11] M.W. Gutowski, J. Nanotechnol., 2011, article ID 952846, doi:10.1155/2011/952846. 